\title{
Comparison of Laughter Yoga and Anti-Anxiety Medication on Anxiety and Gastrointestinal Symptoms of Patients with Irritable Bowel Syndrome
}

\author{
Tahmine Tavakoli ${ }^{1,, \dagger}$, Navid Davoodi ${ }^{1, \dagger}$, Toktam Sadat Jafar Tabatabaee ${ }^{2}$, Zeinab Rostami ${ }^{3}$, \\ Homa Mollaei ${ }^{4}$, Fatemeh Salmani ${ }^{5}$, Sara Ayati ${ }^{1}$, Sanaz Tabrizi ${ }^{1}$
}

1. Department of Gastroenterology, Faculty of Medicine, Birjand University of Medical Sciences, Birjand, Iran

2. Birjand Science and Research Branch, Islamic Azad University, Birjand, Iran

3. Student Research Committee, Birjand University of Medical Sciences, Birjand, Iran

4. Department of Biology, Faculty of Sciences, University of Birjand, Birjand, Iran

5. Social Determinants of Health Research Center, Department of Epidemiology and Biostatistics, Faculty of Health, Birjand University of Medical Sciences, Birjand, Iran

$\dagger$ Both authors contributed equally to this manuscript.

* Corresponding Author:

Tahmine Tavakoli, MD

Department of Internal medicine, Vali-asr Hospital, Birjand, Iran

Tel: +985632443000

Fax: +985632442088

Email: tahminetavakoli95238@yahoo.com

Received: 06 Jun. 2019

Accepted: 09 Sep. 2019

\section{ABSTRACT}

\section{BACKGROUND}

Irritable bowel syndrome (IBS) is the most common chronic gastrointestinal (GI) disorder. Patients with IBS usually suffer from anxiety and depression. A combination of psychological approaches and pharmacological treatments can be a significantly effective treatment for IBS. The main objective of the present study was to provide a therapeutic plan based on laughter yoga and anti-anxiety medication, employed for the very first time, and to determine the effectiveness of these treatments on the anxiety and GI symptoms of patients with IBS.

\section{METHODS}

In this randomized, controlled, clinical trial, the participants were 60 patients selected from those who referred to the GI clinic of Vali-asr Hospital (Birjand, Iran) during the study period (April 2017 to March 2017) and were diagnosed as having IBS based on ROME III criteria. The participants were randomly assigned to either the laughter yoga group, the anti-anxiety medication group, or the symptomatic treatment (control) group. Severity levels of anxiety and GI symptoms before and after intervention were determined and compared among these three groups according to approved protocols.

\section{RESULTS}

The severity of IBS symptoms after the interventions was more greatly reduced in the laughter yoga group than in the anti-anxiety medication and control groups $(p=0.006)$. The severity of anxiety after interventions decreased in all three groups, especially in the yoga treatment group, but the difference was not statistically significant $(p=0.1)$.

\section{CONCLUSION}

Laughter yoga is more effective than anti-anxiety medication in reducing the GI symptoms of patients with IBS. Therefore, applying laughter yoga along with common pharmacological therapies for patients with IBS might be strongly advised.

\section{KEYWORDS:}

Anxiety, Chronic Gastrointestinal Disorder, Gastrointestinal Symptoms, Irritable Bowel Syndrome, Laughter Yoga

\section{Please cite this paper as:}

Tavakoli T, Davoodi N, Jafar Tabatabaee TS, Rostami Z, Mollaei H, Salmani F, Ayati S, Tabrizi S. Comparison of Laughter Yoga and Anti-Anxiety Medication on Anxiety and Gastrointestinal Symptoms of Patients with Irritable Bowel Syndrome. Middle East J Dig Dis 2019;11:211-217. doi:10.15171/mejdd.2019.151.

\section{INTRODUCTION}

Functional gastrointestinal disorders (FGIDs) are more common than digestive disorders, and irritable bowel syndrome (IBS) is the most common chronic 
gastrointestinal (GI) disease, affecting 3-28\% of the population worldwide especially in western countries. It is seen more commonly in women. This disease is influenced by several factors including stress, sleep pattern, and change of environment, and affects a person's daily activities. IBS has bowel symptoms with no organic cause, and it is distinguished by repetitive and inexplicable symptoms like diffuse or localized abdo minal pain, constipation, and diarrhea. ${ }^{1-5}$ IBS symptoms differ among individuals and are characterized by the presence of abdominal pain or bloating and changes in bowel habits with either predominantly diarrhea, constipation, or a combination of both. ${ }^{6}$

IBS has four subtypes: IBS-D (diarrhea), IBS-U (unclassified), IBS-M (mixed, equal diarrhea and constipation types), and IBS-C (constipation), ${ }^{4}$ of them IBS-M is more prevalent than IBS-C and IBS-D. Its pathophysiology is not well known; however, abnormal bowel movements, central nervous system dysfunction, local immune responses to acute infectious gastroenteritis, changes in the gut flora, genetic factors, and mental disorders such as anxiety may be considered as acceptable causes. ${ }^{7,8}$ This heterogeneity in etiology of the disease leads to various symptoms in affected patients.

Lifestyle changes, dietary advices, psychological medications, and agents that target GI motility have been used for managing IBS.

IBS has a strong body-mind association. Patients with IBS usually have poor mental health, and more than $70-90 \%$ of such patients also suffer from anxiety and depression. A combination of psychotherapy with traditional pharmaceutical procedures or psychotherapy alone is approved approaches to the treatment of IBS. Among the psychotherapeutic interventions, behavioral therapy is more commonly recommended. ${ }^{9}$

Complementary treatments are generally accepted, because they have low costs, and fewer side effects. One new strategy is the performance of laughter exercises known as laughter yoga (laughter with yoga breathing and stretching exercises; people should laugh with no joke or comic program), which has been developed by Kataria (Indian physician). Kataria believes that both types of laughter, artificial and natural, can have similar effects on the body. ${ }^{10,11}$ Laughter therapy has been widely shown to have a positive impact on physical and mental health, such as changes to the immune system, muscular comfort, hormones, and the mind. ${ }^{12-14}$ In the current study we aimed to compare the effects of laughter yoga with those of anti-anxiety medications on the anxiety and GI symptoms of patients with IBS.

\section{MATERIALS AND METHODS}

The design of this study was a randomized, controlled, clinical trial with pre-test and post-test evaluations. Patients were selected from those who referred to the GI clinic of Vali-asr Hospital (Birjand, Iran) during the study period (April 2017 to March 2017) and were diagnosed as having IBS based on ROME III criteria. Using the convenience sampling method, 60 participants were selected randomly from those meeting the inclusion criteria for this study. The inclusion criteria were: a diagnosis of IBS based on the mentioned IBS protocol, the age range of 18-50 years, willingness to participate after the research objectives and the advantages and disadvantages of the methods were explained, and willingness and ability to maintain the fixed type and dose of drug (in cases of drug therapy). To consider ethical principles, written consent to participate was obtained from each patient. Exclusion criteria comprised non-compliance with medicinal recommendations, lack of regular attendance in classes, using psychological treatments before participating in the study, suffering from other structural GI or functional diseases during the study period, psychotic disorders, or personality disorders, and using anti-anxiety drugs before starting the study. The participants were assigned to one of three groups with 20 participants in each: group A (laughter yoga therapy), group B (anti-anxiety medication), or group C (symptomatic therapy only). Data were collected in two parts: measuring the scale of severity of IBS symptoms and Beck's Anxiety Inventory.

For data analysis, SPSS software version 23 was used. After data were collected, a file was created in the SPSS software. The variables of severity of symptoms and anxiety had an abnormal distribution. Hence, to compare the mean values of severity of symptoms and anxiety among the three groups, the Kruskal Wallis and Mann Whitney tests were used. The Wilcoxon test was also used for each group before and after intervention. The variable age showed a normal distribution, and ANOVA was used to compare the mean age ranges of the three groups. A $p$ value of 0.05 was considered as statistically significant. 
Table 1: Summary of educational package of laughter yoga

\begin{tabular}{lll}
\hline \multirow{2}{*}{ Session } & \multicolumn{1}{c}{ Topic } & Time \\
\hline \multirow{2}{*}{2} & Step 1: Introducing principles and rules of laughter yoga & $20 \mathrm{~min}$ \\
\cline { 2 - 3 } & Step 2: Familiarity and introduction & $20 \mathrm{~min}$ \\
\cline { 2 - 3 } & Step 3: Defining laughter yoga for participants & $20 \mathrm{~min}$ \\
\hline \multirow{2}{*}{3} & Step 1: Introducing advantages of laughter & $25 \mathrm{~min}$ \\
\cline { 2 - 3 } & Step 2: Psychological and emotional impacts of laughter & $35 \mathrm{~min}$ \\
\hline \multirow{2}{*}{4} & Step 1: Defining yoga & $30 \mathrm{~min}$ \\
\hline \multirow{2}{*}{5} & Step 2: Importance of deep breathing & $30 \mathrm{~min}$ \\
\cline { 2 - 3 } & Step 1: Value laughter techniques & $25 \mathrm{~min}$ \\
\hline \multirow{2}{*}{ Step 2: Cheerful laughter techniques } & $35 \mathrm{~min}$ \\
\cline { 2 - 3 } & Step 1: Method of implementing laughter yoga & $25 \mathrm{~min}$ \\
\cline { 2 - 3 } & Step 2: Introducing steps of implementing laughter yoga & $20 \mathrm{~min}$ \\
\hline Step 3: Introducing types of laughter & $20 \mathrm{~min}$ \\
\hline 7 & Step 1: Defining meditation & $10 \mathrm{~min}$ \\
\cline { 2 - 3 } & Step 2: Introducing general framework for laughter meditation & $15 \mathrm{~min}$ \\
\cline { 2 - 3 } & Step 3: Defining relaxation & $15 \mathrm{~min}$ \\
\cline { 2 - 3 } & Step 4: Relaxation steps & $20 \mathrm{~min}$ \\
\hline
\end{tabular}

\section{Research Tools}

\section{Demographic Information}

The demographic characteristics of the patients comprised sex, age, type of diagnosed disease, and disease history.

\section{ROME-III Diagnostic Criteria for IBS}

The ROME-III questionnaire was used as the standard scale for IBS assessment with a high credibility. ${ }^{15}$

\section{IBS Symptom Severity Scale (IBS-SSS)}

The IBS Symptom Severity Index presented by Francis CY and colleagues (1997) was used in this study. It has also been used in similar studies. This instrument contains five items, each scored in a range of 0-5 for the 10-day period prior to the study. A score of 8-18 indicated mild severity, 18-30 indicated medium severity, and a score higher than 30 indicated a severe level of symptoms. ${ }^{16}$

\section{Beck's Anxiety Questionnaire}

Beck's Anxiety Questionnaire was introduced by Beck and co-workers in $1996{ }^{17}$ and contains 21 items for measuring anxiety symptoms in patients over a 2-week period. Each item in this questionnaire is scored based on a 4 -point Likert scale $(0=$ never to $3=$ severe to intolerable level). ${ }^{17,18}$ The reliability test of Beck's Anxiety Questionnaire among Iranian samples showed that the total reliability coefficient of the questionnaire equaled $0.91 .^{19}$

\section{Intervention Protocols}

Participants were asked to attend an explanatory session in which the research objectives were explained. All participants in all three groups (laughter yoga therapy, anti-anxiety medication, and symptomatic therapy only) completed the relevant scales before beginning the experimental interventions in the pretest step, immediately after entering the study, and again at the end of interventions in the post-test step to measure the effects of the interventions.

\section{Laughter Yoga Therapy Protocol}

The laughter yoga intervention was the same for all patients in this group. It was implemented in seven sessions by a teacher familiar with this method (table 1).

\section{Anti-anxiety Therapy Protocol}

Participants in the anti-anxiety medication group were given sertraline at a dose of 50 to $200 \mathrm{mg}$ per day.

\section{Symptomatic Therapy Protocol}

Because of the differences in symptom severity among patients, it was impossible to synchronize the 
Table 2: Comparing the anxiety scores and severity of IBS symptoms between the three groups using ANOVA test

\begin{tabular}{|c|c|c|c|c|c|}
\hline & Yoga & Anti-anxiety medication & Control & $\mathbf{F}$ & $P$ value \\
\hline BECK before & $20.8 \pm 8.1$ & $19 \pm 9.1$ & $20.7 \pm 9.3$ & 0.23 & 0.78 \\
\hline BECK after & $13.3 \pm 4.6$ & $13.3 \pm 4.2$ & $17 \pm 8.3$ & 2.39 & 0.1 \\
\hline BECK difference & $7.5 \pm 4.06$ & $5.7 \pm 7.09$ & $3.7 \pm 4.15$ & 3.14 & 0.05 \\
\hline IBS before & $27.8 \pm 6.4$ & $28.7 \pm 7.8$ & $29.2 \pm 7.4$ & 0.176 & 0.83 \\
\hline IBS after & $15.6 \pm 5.4$ & $18.6 \pm 5.8$ & $22.3 \pm 6.7$ & 5.73 & 0.006 \\
\hline IBS difference & $12.2 \pm 4.43$ & $10.1 \pm 8.46$ & $6.9 \pm 2.79$ & 4.061 & 0.023 \\
\hline
\end{tabular}

IBS: Irritable bowel syndrome

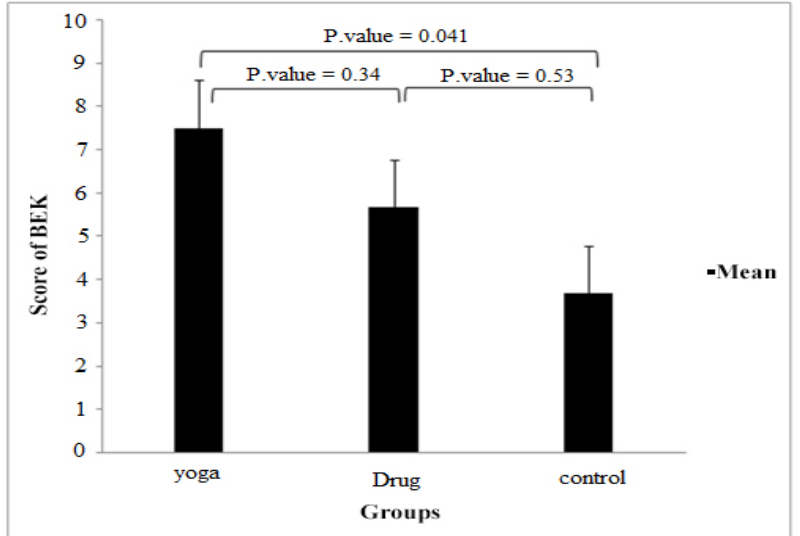

Fig.1: Changes in anxiety scores in the three treatment groups

drug dose, and the intervention was not the same in the symptomatic treatment group. However, no patient in this group received anti-anxiety drugs.

\section{RESULTS}

In the present study, 60 patients with IBS diagnosed based on ROME-III were assigned to three different treatment groups: the laughter yoga treatment group, the anti-anxiety medication group, or the symptomatic treatment (control) group, of whom 19, 18, and 18 patients, respectively, completed the study. One person left the laughter yoga group because follow-up was not possible; two cases were dropped out from the anti-anxiety medication group because of drug side effects, and two patients were excluded from the symptomatic treatment group because follow-up was not possible. The groups were homogeneous in age $(p=0.902)$, sex $(p=1)$, and pre-test IBS severity score $(p=0.839)$. The mean age of the patients was 33.10 (9.49), 32.38 (9.23), and 31.72 (9.02) years in the laughter yoga group, anti-anxiety medication group, and control group, respectively. Most of the participants were female $(n=44,80 \%)$ and the scores of

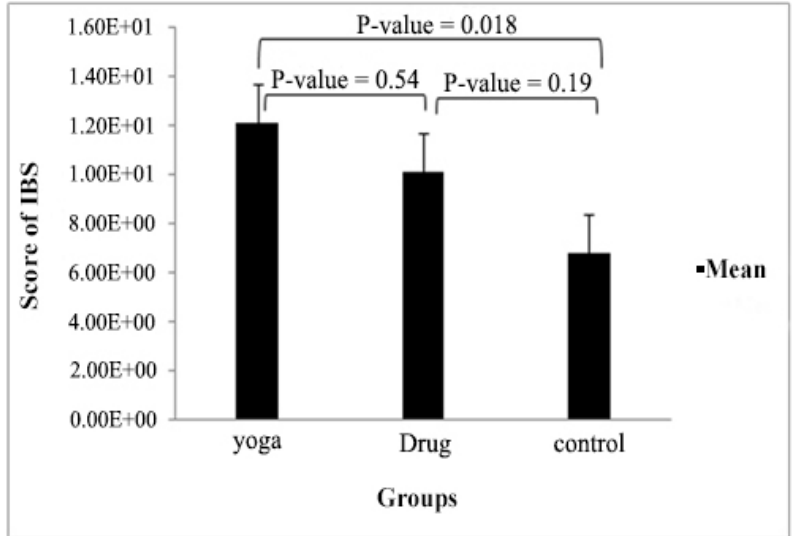

Fig.2: Changes in IBS scores in the three treatment groups

anxiety and IBS before intervention were homogenous in all groups.

The results showed that there was no significant difference between the mean values of anxiety in the three groups either before intervention $(p=0.78)$ or after intervention $(p=0.1)$. However, a significant difference was observed in the Beck scores of before and after intervention ( $p=0.05$ ) (table 2). The results also showed no significant difference between the mean values of IBS symptoms in the three groups before intervention ( $p=0.83$ ). However, the severity of IBS symptoms score was significantly different between the yoga and meditation groups compared with the controls after intervention $(p=0.006)$. A significant difference was also seen in the IBS scores of before and after intervention $(p=0.023$, table 2).

Finally, assessing the changes in anxiety and IBS scores in the three treatment groups with the follow-up test (Tukey) indicated that the participants in the laughter yoga group showed more significant differences (figures 1 and 2). Significant differences between the laughter yoga and control groups were also seen in changes to 
Table 3: Results of linear regression model containing intervention groups and BECK scores

\begin{tabular}{|c|c|c|c|c|c|}
\hline \multirow{2}{*}{ Variables } & \multicolumn{2}{|c|}{ Unstandardized coefficients } & \multirow{2}{*}{$\begin{array}{c}\text { Standardized coefficients } \\
\text { Beta }\end{array}$} & \multirow{2}{*}{$\mathbf{t}$} & \multirow{2}{*}{$p$ value } \\
\hline & $\mathbf{B}$ & Std. Error & & & \\
\hline Intercept & 13.465 & 2.397 & & 5.617 & $<0.001$ \\
\hline Yoga & -4.72 & 1.772 & -0.35 & -2.663 & 0.01 \\
\hline Drug & -1.83 & 1.792 & -0.13 & -1.021 & 0.312 \\
\hline BECK after & 0.523 & 0.121 & 0.491 & 4.334 & $<0.001$ \\
\hline
\end{tabular}

IBS scores $(p=0.018)$ and Beck scores $(p=0.041)$.

Based on the linear regression model, the average IBS score in the laughter yoga group decreased 0.35 units and in the medication group decreased 0.13 units compared with the control group. This result indicated the greater effect of the laughter yoga intervention on reducing IBS symptoms. Also, with a one-unit increase in the anxiety score, the IBS score increased an average of 0.49 units (table 3).

\section{DISCUSSION}

IBS is the most common chronic GI disease and is highly correlated with psychological factors, like depression and anxiety. Because of the psychological pathology of the disease, considering psychological approaches and non-pharmacological treatments can be significantly effective in reducing symptoms as well as treatment costs of the disease. Among psychotherapeutic interventions, behavioral therapy is more commonly recommended. ${ }^{2}$ In the current study, the effects of laughter yoga and antianxiety medication on the anxiety and GI symptoms of patients with IBS were compared.

Studies have shown that laughing can increase brain endorphins, and 5 minutes of laughing can relieve pain for 5 hours. ${ }^{20}$ Berk and colleagues studied the changes in neuroendocrine components and stress hormones in response to laughing. Their results showed that while cortisol decreased faster in the experimental group, epinephrine significantly increased in this group and then decreased with laughing. ${ }^{20}$

The present study is a unique work that compared the methods of anti-anxiety medication and laughter yoga separately. In previous studies on the effects of psychological treatment on IBS, only combined treatments were compared.

Most of the patients in this study were female, indicating a higher prevalence of the disease among women.
Other studies have confirmed this finding. ${ }^{5,21}$ The current results indicated that after the designed interventions, the severity of IBS symptoms was more greatly reduced in the laughter yoga group, and the observed differences were statistically significant ( $p=0.006$ ). After intervention, the severity of anxiety was changed in all three groups; the greatest reduction was observed in the laughter yoga group, but the observed differences were not statistically significant ( $p=0.1$, which may be due to the small sample size.

The findings are in accordance with those of previous studies. Keykha and co-workers studied the effects of 8 weeks laughter yoga exercises on motor function, balance, and flexibility in patients with Parkinson's disease. The results showed a significant improvement $(p<0.05)$ in the motor function of patients after 8 weeks of intervention, and laughter yoga was recommended as a useful modality for such patients. ${ }^{22}$

Mahdipoor and colleagues investigated the effects of psychological and pharmacological treatments on the reduction of symptoms and improvement of mental health and quality of life in patients with IBS. Their results showed that this combination therapy could increase mental health, improve quality of life, and reduce symptoms in patients with IBS. ${ }^{19}$ Moreover, in studies conducted by Greed and colleagues and Drossman and others, the combined treatment of psychological therapy and antidepression drugs was effective against IBS. ${ }^{23,24}$

The present study showed good short-term (7-week period) results, but determining long-term effects was impossible because of ethical considerations. In another study, Lackner and colleagues studied the rapid response of cognitive-behavioral therapy (CBT) to symptoms of patients with IBS. Their results showed a fast reduction in symptoms after a 4-week period of CBT interventions. ${ }^{25}$ Solati, Dehkordi, and Adibi assessed the effects of CBT along with pharmacological treatment on quality of life, 
frequency, and severity of symptoms in patients with IBS. They found that the mean value of quality of life was significantly higher in the CBT and pharmacological treatment group than in the pharmacological group in both pre- and post-treatment steps, although no significant difference was observed in the follow-up. ${ }^{21}$ This finding contradicted those of previous studies that showed CBT and medical treatment had no effect on the frequency and severity of symptoms in patients with IBS over a 6-month period, because after the last intervention, patients experienced a relapse of the severity of symptoms. ${ }^{26,27}$

Although several studies have reported positive effects of anti-depressants in reducing symptoms and improving the quality of life of patients with IBS,,$^{28,29}$ which are in consistency with our findings, some studies have shown that anti-depressants (citalopram and amitriptyline) are not better than placebos in reducing the symptoms of such patients. ${ }^{30,31}$ Such result might be explained by the lack of a proper design or the effect of disrupting factors.

In conclusion, the current results have shown that laughter yoga is more effective than anti-anxiety medication in reducing GI symptoms in patients with IBS and in improving the quality of life of these patients. Thus, it is strongly recommended that laughter yoga be coupled with common pharmacological therapies for IBS.

\section{Limitations}

The most important limitation of this study was the absence of a long-term follow-up of the patients due to ethical issues. Because of the limited number of laughter yoga sessions, the impossibility of continuing these sessions, and the chronic nature of the disease after the 7-week course, another method should be applied. Moreover, the lack of separation between IBS patients with diarrhea (IBS-D) and those with constipation (IBS-C) who require different symptomatic treatments was another limitation of this study.

\section{ETHICAL APPROVAL}

There is nothing to be declared.

\section{CONFLICT OF INTEREST}

The authors declare no conflict of interest related to this work.

\section{REFERENCES}

1. Iftikhar B, Naveed H, Khalil K, ur Rehman H, Khushbaght. Prevalence of Irritable Bowel Syndrome and it's Risk Factors Among Medical Students of Peshawar, Pakistan. Pakistan J Med Res 2018;57:24-8.

2. Klem F, Wadhwa A, Prokop LJ, Sundt WJ, Farrugia G, Camilleri M, et al. Prevalence, risk factors, and outcomes of irritable bowel syndrome after infectious enteritis: a systematic review and meta-analysis. Gastroenterology 2017;152:1042-54. doi: 10.1053/j.gastro.2016.12.039.

3. Williams M. Irritable bowel syndrome: the latest thinking. J Community Nursing 2018;32:28-33.

4. Markland AD, Jelovsek JE, Rahn DD, Wang L, Merrin $\mathrm{L}$, Tuteja A, et al. Irritable bowel syndrome and quality of life in women with fecal incontinence. Female Pelvic Med Reconstr Surg 2017;23:179-83. doi: 10.1097/ SPV.0000000000000358.

5. Sunderland R. Irritable bowel syndrome in adults: symptoms, treatment and management. Nurs Stand 2017;31:52-63. doi: 10.7748/ns.2017.e10654.

6. McCullough R, McCullough J. Deciphering the pathophysiology of irritable bowel syndrome and functional gastrointestinal disorders-an alternative model for pathogenesis: cytokine controlled transepithelial multi-feedback loop. Transl Gastroenterol Hepatol 2017;2:18. doi: $10.21037 / \operatorname{tgh} .2017 .03 .02$

7. Drossman DA, Hasler WL. Rome IV-functional GI disorders: disorders of gut-brain interaction. Gastroenterology 2016;150:1257-61. doi: 10.1053/j.gastro.2016.03.035.

8. Kawoos Y, Wani ZA, Kadla SA, Shah IA, Hussain A, Dar MM, et al. Psychiatric Co-morbidity in Patients With Irritable Bowel Syndrome at a Tertiary Care Center in Northern India. J Neurogastroenterol Motil 2017;23:55560. doi:10.5056/jnm16166.

9. Lackner JM, Ma CX, Keefer L, Brenner DM, Gudleski GD, Satchidanand N, et al. Type, rather than number, of mental and physical comorbidities increases the severity of symptoms in patients with irritable bowel syndrome. Clin Gastroenterol Hepatol 2013;11:1147-57. doi: 10.1016/j.cgh.2013.03.011.

10. Yazdani M, Esmaeilzadeh M, Pahlavanzadeh S, Khaledi F. The effect of laughter yoga on general health among nursing students. Iran J Nurs Midwifery Res 2014;19:36-40.

11. DeCaro DS, Constantine Brown JL. Laughter Yoga, Adults Living With Parkinson' s Disease, and Caregivers: A Pilot Study. Explore (NY) 2016;12:196-9. doi: 10.1016/j.explore.2016.02.005

12. Shahabizade F, Hassanzadeh-Taheri M, Fatemi S, Moodi $\mathrm{H}$, Hosseini M, Akbari A, et al. Evaluating the impact of laughter therapy on hope, self-efficacy, and pain control in patients with chronic low back pain. Pharmacophore 2017; 8: e-1173662.

13. Umamah F, Mufarrihah N. Pengaruh terapi tertawa terhadap tingkat kecemasan pada lanjut usia di uptd griya werdha surabaya. J Health Sci 2018;11. doi: 10.33086/jhs.v11i1.116. 
14. Briggs JP, Longo DL, Fauci AS, Kasper DL, Hauser SL, Jameson JL, Loscalzo J. Complementary, Alternative, and Integrative Health Practices. Harrison's principles of internal medicine 2015.

15. Francis CY, Morris J, Whorwell PJ. The irritable bowel severity scoring system: a simple method of monitoring irritable bowel syndrome and its progress. Aliment Pharmacol Ther 1997;11:395-402. doi: 10.1046/j.13652036.1997.142318000.x.

16. Beck AT, Steer RA, Ball R, Ranieri W. Comparison of Beck Depression Inventories-IA and-II in psychiatric outpatients. J Pers AssessC1996;67:588-97.

17. Dabson K, Mohammad KP. Psychometric characteristics of Beck depression inventory-II in patients with major depressive disorder. J Pers Assess 1996;67:588-97. doi: 10.1207/s15327752jpa6703 13.

18. Mahdipour MZ, Davoudi A. Comparison of the Effectiveness of Psychological-Pharmaceutical Combined Therapy with Drug Therapy on Mental Health, Improving Quality of Life and Symptoms Reduction in Patients with Irritable Bowel Syndrome in Ahwaz. 2012; 3.

19. Weisse AB. editor Humor in medicine: Can laughter help in healing? Proc (Bayl Univ Med Cent) 2017;30: 378-81. doi: 10.1080/08998280.2017.11929656.

20. Greenberg M AH, Galiczynski E. A contemporary review of IBS. Phys Assist 2002; 26: 26-33.

21. Solati Dehkordy K, Adibi P, Ghamarani A. The effects of cognitive-behavior therapy and drug therapy on quality of life and symptoms of patients with irritable bowel syndrome. J Kerman Uni Med Sci 2012;19.

22. Hussein poor A EN, Chitaz A. The effect of eight weeks of laughing yoga exercises on motor function, balance and flexibility of patients with Parkinson's disease. Res Rehabilit Sci 2013;9:39-47.

23. Creed F, Fernandes L, Guthrie E, Palmer S, Ratcliffe J, Read N, et al. The cost-effectiveness of psychotherapy and paroxetine for severe irritable bowel syndrome. Gastroenterology 2003;124: 303-17. doi: 10.1053/ gast.2003.50055.

24. Drossman D, Morris CB, Hu Y, Toner BB, Diamant N, Whitehead WE, et al. Characterization of health related quality of life (HRQOL) for patients with functional bowel disorder (FBD) and its response to treatment. $\mathrm{Am}$ $J$ Gastroenterol 2007;102:1442-53. doi: 10.1111/j.15720241.2007.01283.x.

25. Lackner JM, Gudleski GD, Keefer L, Krasner SS, Powell C, Katz LA. Rapid response to cognitive behavior therapy predicts treatment outcome in patients with irritable bowel syndrome. Clin Gastroenterol Hepatol 2010; 8:426-32. doi: 10.1016/j.cgh.2010.02.007.

26. Camilleri M, Northcutt AR, Kong S, Dukes GE, McSorley D, Mangel AW. Efficacy and safety of alosetron in women with irritable bowel syndrome: a randomised, placebocontrolled trial. Lancet 2000;355:1035-40. doi: 10.1016/ S0140-6736(00)02033-X.
27. Moss-Morris R, McAlpine L, Didsbury L, Spence M. A randomized controlled trial of a cognitive behavioural therapy-based self-management intervention for irritable bowel syndrome in primary care. Psychol Med 2010;40:85-94. doi: 10.1017/S0033291709990195.

28. Tack J, Broekaert D, Fischler B, Van Oudenhove L, Gevers AM, Janssens J. A controlled crossover study of the selective serotonin reuptake inhibitor citalopram in irritable bowel syndrome. Gut 2006;55:1095-103. doi: 10.1136/gut.2005.077503.

29. Vahedi H, Merat S, Momtahen S, Kazzazi A, Ghaffari N, Olfati G, et al. Clinical trial: the effect of amitriptyline in patients with diarrhoea-predominant irritable bowel syndrome. Aliment Pharmacol Ther 2008;27:678-84. doi: 10.1111/j.1365-2036.2008.03633.x.

30. Ladabaum U, Sharabidze A, Levin T, Zhao W, Chung E, Bacchetti P, et al. Citalopram is not-effective therapy for non-depressed patiants with irritabl bowel syndrome. Clin Gastroenterol Hepatol 2010;81:42.

31. Talley NJ, Kellow JE, Boyce P, Tennant C, Huskic $\mathrm{S}$, Jones M. Antidepressant therapy (imipramine and citalopram) for irritable bowel syndrome: a doubleblind, randomized, placebo-controlled trial. Dig Dis Sci 2008;53:108-15. doi: 10.1007/s10620-007-9830-4. 